\title{
Calibration of a hand-held haemoglobin analyser for use on fish blood
}

\author{
T. D. Clark*†, E. J. Eliason*, E. Sandblom \\ AND A. P. FARRELL* \\ *Faculty of Land and Food Systems, University of British Columbia, Vancouver, V6T 1 Z4 \\ Canada, \$Department of Zoology, University of Gothenburg, Box 463, S-405 30 \\ Gothenburg, Sweden and §Department of Forest Sciences, and The Institute for \\ Resources, Environment and Sustainability, University of British Columbia, Vancouver, \\ V6T 1Z4 Canada
}

(Received 4 April 2008, Accepted 25 September 2008)

\begin{abstract}
The HemoCue haemoglobin analyser consistently overestimated haemoglobin concentration $([\mathrm{Hb}])$ in the blood of all fish species (sockeye salmon Oncorhynchus nerka, Chinook salmon Oncorhynchus tshawytscha, Pacific bluefin tuna Thunnus orientalis and chub mackerel Scomber japonicus) by $22-50 \%\left(9 \cdot 9-36 \cdot 0 \mathrm{~g} \mathrm{l}^{-1}\right)$ over a $\left[\mathrm{Hb}\right.$ ] range of $20-160 \mathrm{~g} \mathrm{l}^{-1}$. The systematic nature of the overestimation, however, allowed the formulation of an accurate calibration equation that can be used to correct values of $[\mathrm{Hb}]$ measured by the HemoCue in field studies. (c) 2008 The Authors Journal compilation $\odot 2008$ The Fisheries Society of the British Isles
\end{abstract}

Key words: Drabkin's method; haemoglobin concentration; haemoglobinometer; HemoCue $\mathrm{Hb}$ $201^{+}$; spectrophotometer.

Blood haemoglobin concentration ([Hb]) can be measured on microlitre blood samples and has been used widely with many vertebrates to provide a snapshot of general physiological condition (Frisch \& Anderson, 2005; Artacho et al., 2007; Banbura et al., 2007; Imsland et al., 2007), and an index of such things as oxygen carrying capacity (Ganong, 1973; Lay \& Baldwin, 1999; Burness et al., 2000) and haematophagous parasite intensity (O'Brien et al., 2001; Dudaniec et al., 2006). The most common and arguably the most accurate method of determining [Hb] is Drabkin's method (Drabkin \& Austin, 1935; Balasubramaniam \& Malathi, 1992), which estimates [Hb] spectrophotometrically by mixing a small blood sample with a solution containing a cyanide derivative (Drabkin's reagent). Drabkin's reagent haemolyses the erythrocytes and all haemoglobin derivatives are oxidized by ferricyanide to produce methaemoglobin. Methaemoglobin and cyanide form a stable

†Author to whom correspondence should be addressed. Tel.: +1 604822 9986; fax: +1 604822 2184; email: timothy.clark.mail@gmail.com 
cyanomethaemoglobin complex, the absorbance of which is used to calculate $[\mathrm{Hb}]$. The Drabkin method, however, is laborious and is poorly suited to field situations given the need for a cyanide solution and a spectrophotometer. As such, field biologists typically store blood samples with an anticoagulant for subsequent analysis of [Hb], precluding rapid assessment of interindividual physiological status of perhaps large populations, such as schooling fishes. Thus, a more portable and practical method for determining [Hb] clearly would be beneficial to fish biologists to negate the need for laboratory-based [ $\mathrm{Hb}]$ analyses. The advent of hand-held [Hb] analysers for the medical industry offers a possible solution to this problem.

The present study was concerned with the use of the HemoCue ${ }^{\mathrm{TM}}$ haemoglobin analyser (HemoCue $201^{+}$, Ängelholm, Sweden) to assess fish blood samples. This analyser has been proven accurate for mammalian blood (von Schenck et al., 1986; Lardi et al., 1998) and has been used to measure [Hb] of birds and fishes, but without validation (Alvarez-Pellitero \& Pinto, 1987; Nyholm et al., 1995; Burness et al., 2000; Schmitt et al., 2005; Dudaniec et al., 2006; Eklom \& Lill, 2006; Simmons \& Lill, 2006; Schmitt et al., 2007). The present study examined the accuracy of the HemoCue analyser in determining $[\mathrm{Hb}]$ of fish blood by performing simultaneous measurements using the HemoCue and the Drabkin method as a reference standard. Four fish species were examined: sockeye salmon Oncorhynchus nerka (Walbaum), Chinook salmon Oncorhynchus tshawytscha (Walbaum), Pacific bluefin tuna Thunnus orientalis (Temminck \& Schlegel) and chub mackerel Scomber japonicus Houttuyn.

Adult $O$. tshawytscha $(2-5 \mathrm{~kg} ; n=7)$ and $O$. nerka $(2-3 \mathrm{~kg} ; n=8)$ were collected from the lower Fraser River and its tributaries, British Columbia, Canada. These fishes were kept in 100001 tanks for $1-4$ weeks at $13-22^{\circ} \mathrm{C}$ for use in experiments described elsewhere (e.g. Clark et al., 2008). Blood samples for the present study were obtained either from caudal venepuncture or from catheters placed in the dorsal aorta and sinus venosus. Samples were collected from live or freshly killed fishes into heparinized syringes or ethylenediaminetetra-acetic acid (EDTA)-coated vacutainers, and either analysed immediately or after refrigeration at $4^{\circ} \mathrm{C}$ for $<20 \mathrm{~h}$.

Juvenile $T$. orientalis (10-15 $\mathrm{kg} ; n=5)$ and adult $S$. japonicus $(0 \cdot 5-1 \cdot 5 \mathrm{~kg}$; $n=5)$ were utilized at the Tuna Research and Conservation Centre, Monterey Bay, U.S.A. Fishes were housed in $25000-1000001$ tanks at $17-20^{\circ} \mathrm{C}$ and were sampled immediately after being sacrificed for another series of experiments. Blood from the tail vein or from the heart was collected into heparinized syringes or EDTA-coated vacutainers, and then analysed immediately or after refrigeration at $4^{\circ} \mathrm{C}$ for $<10 \mathrm{~h}$.

Haemoglobin concentration was measured on whole blood of each species. Additionally, sub-samples of whole blood were either haemo-diluted once or twice with saline $(0.6 \% \mathrm{NaCl})$, or haemo-concentrated by centrifuging at $10000 \mathrm{~g}$ prior to removal of some plasma and subsequent re-suspension of erythrocytes in the remaining plasma. Both Drabkin's method and the HemoCue method were used to analyse each blood sample (the mean of triplicate samples was used in all cases). Human blood (TDC) was used as a control 
to verify correct measurements of [Hb] for both methods. Human blood was haemo-diluted and haemo-concentrated as above.

Drabkin's reagent was made using a commercially available kit (HG 980; Randox Laboratories, Crumlin, U.K.). Aliquots of $1 \mathrm{ml}$ Drabkin's reagent were placed into Eppendorf tubes, covered with foil and stored in a refrigerator at $4^{\circ}$ C. Mixed blood samples of $10 \mu \mathrm{l}$ were placed into the $1 \mathrm{ml}$ aliquots of Drabkin's reagent as required, immediately mixed using a vortex and then allowed to incubate at room temperature for up to $1 \mathrm{~h}$ with frequent agitation. Following the incubation period, samples were centrifuged at $10000 \mathrm{~g}$ for $10 \mathrm{~min}$ to separate out degraded proteins. The supernatant was transferred into spectrophotometer vials for subsequent analysis of absorbance at $540 \mathrm{~nm}$. Absorbance was measured within $30 \mathrm{~min}$ and several times within the following $5 \mathrm{~h}$.

The absorbance of the solution was converted to [Hb] $\left(\mathrm{g}^{-1}\right)(y)$ according to: $y=\left(A_{540}-A_{\mathrm{BLANK}}\right) W_{\mathrm{Hb}} F_{\mathrm{D}}\left(C_{\mathrm{E}} d 1000\right)^{-1}$, where $A_{540}$ is the absorbance at $540 \mathrm{~nm}, A_{\mathrm{BLANK}}$ is the absorbance of pure Drabkin's reagent at $540 \mathrm{~nm}$, $W_{\mathrm{Hb}}$ is the molecular mass of the human haemoglobin tetramer (=64 458), $F_{\mathrm{D}}$ is the dilution factor for $10 \mu \mathrm{l}$ blood in $1 \mathrm{ml}$ Drabkin's reagent $(=101), C_{\mathrm{E}}$ is the millimolar extinction coefficient of tetrameric cyanomethaemoglobin at 540 $\mathrm{nm}(=44), d$ is the vial light path in $\mathrm{cm}(=1)$ and 1000 converts from $\mathrm{mg}$ to $\mathrm{g}$ (adapted from Dacie \& Lewis, 1975; ICSH, 1978). More simply, $A_{540}-A_{\text {BLANK }}$ was multiplied by 148 .

The HemoCue $\mathrm{Hb} \mathrm{201}{ }^{+}$analyser $(\mathrm{L} \times \mathrm{W} \times \mathrm{D}=160 \times 85 \times 43 \mathrm{~mm}$; www.hemocue.com) weighs $350 \mathrm{~g}$ and can be powered by an AC power adaptor or type AA batteries. It has a measurement range of $0-256 \mathrm{~g}^{-1}$ and the technical specifications report a coefficient of variance (c.v.) for human blood of $<2 \%$ in comparison with the cyanomethaemoglobin method. Its robustness is equivalent to a cellular phone, and it can be purchased with a padded carry case $(\mathrm{L} \times \mathrm{W} \times \mathrm{D}=260 \times 220 \times 70 \mathrm{~mm})$ for added protection under field conditions. The HemoCue technique is based on an optical measuring cuvette $(\mathrm{L} \times \mathrm{W} \times \mathrm{D}=35 \times 15 \times 2 \mathrm{~mm})$ of small volume $(10 \mu \mathrm{l})$ and short light path $(0.13 \mathrm{~mm}$ distance between the parallel walls of the optical window). Dry reagents are deposited on the inner walls of the cuvette cavity, and the blood sample, drawn into the cavity by capillary action, is mixed with the reagents spontaneously. The erythrocyte membranes are lysed by sodium deoxycholate, sodium nitrite converts haemoglobin iron from the ferrous to the ferric state to form methaemoglobin, and then methaemoglobin combines with azide to form azide methaemoglobin (Vanzetti, 1966; von Schenck et al., 1986). The cuvette is given 1-10 $\mathrm{min}$ at room temperature to allow chemical reactions to take place (refer to product user manual), and then it is inserted into the HemoCue analyser where a built-in photometer measures its absorbance at 570 and $880 \mathrm{~nm}$ (the use of a double wavelength measuring method compensates for blood turbidity). The analyser calculates the concentration of haemoglobin in the sample and displays the result. In the present study, all samples were run in triplicate (i.e. three cuvettes per sample of each haemoglobin concentration), and each cuvette was placed in the analyser 1-2, 3-4, 7-8 and 9-11 min after being filled with blood, to monitor any change in the measured [Hb] with increasing cuvette incubation time. 
Values of [Hb] for each blood sample were calculated as the mean of triplicate samples. The variance in the triplicate samples measured by the HemoCue ranged from $0 \cdot 0$ to $7 \cdot 6 \%$ (mean $2 \cdot 3 \%$ ), and that of the Drabkin method ranged from $0 \cdot 4$ to $15 \cdot 8 \%$ (mean $4 \cdot 4 \%$ ). Mean values are used here. Least-squares regression was used to formulate relationships between the Drabkin and HemoCue methods. The Johnson-Neyman technique (White, 2003) was used in Fig. 1 to determine if there existed a range of $x$ values for which there was no difference between the regression and line of equality. Significance was considered at $P<0.05$.

Using Drabkin method as the reference standard, the HemoCue analyser accurately measured [Hb] of human blood (Fig. 1). A strong linear relationship existed between the results for the HemoCue ([Hb] HEMO) and Drabkin $\left([\mathrm{Hb}]_{\mathrm{DRAB}}\right)$ methods, but the HemoCue analyser significantly overestimated $[\mathrm{Hb}]$ for fishes of all species at all haemoglobin concentrations $(P<0.05$; Johnson-Neyman technique). The overestimation ranged from $50 \%(9.9 \mathrm{~g}$ $\left.1^{-1}\right)$ at $[\mathrm{Hb}]_{\text {DRAB }}$ of $20 \mathrm{~g}^{-1}$ to $22 \%\left(36.0 \mathrm{~g} \mathrm{l}^{-1}\right)$ at $[\mathrm{Hb}]_{\text {DRAB }}$ of $160 \mathrm{~g} \mathrm{l}^{-1}$. For fish blood, $[\mathrm{Hb}]$ measured by the Drabkin method was linearly related to [Hb] measured by the HemoCue method (Fig. 1), where $P<0.001$ and $r^{2}$ $=0.967$. Thus, the second equation in the legend to Fig. 1 can be used to correct fish $[\mathrm{Hb}]$ measured with the HemoCue.

Although the measurement of $[\mathrm{Hb}]_{\text {HEMO }}$ for the blood of human, S. japonicus and $T$. orientalis varied little with increasing cuvette incubation time $(<3 \%$ change; Fig. 2), that of $O$. nerka and $O$. tshawytscha typically increased by $7 \%$ throughout the $11 \mathrm{~min}$ incubation period (Fig. 2). In contrast, measurements obtained using the Drabkin method remained stable for up to $5 \mathrm{~h}$ while the solutions in the spectrophotometer vials incubated at room temperature.

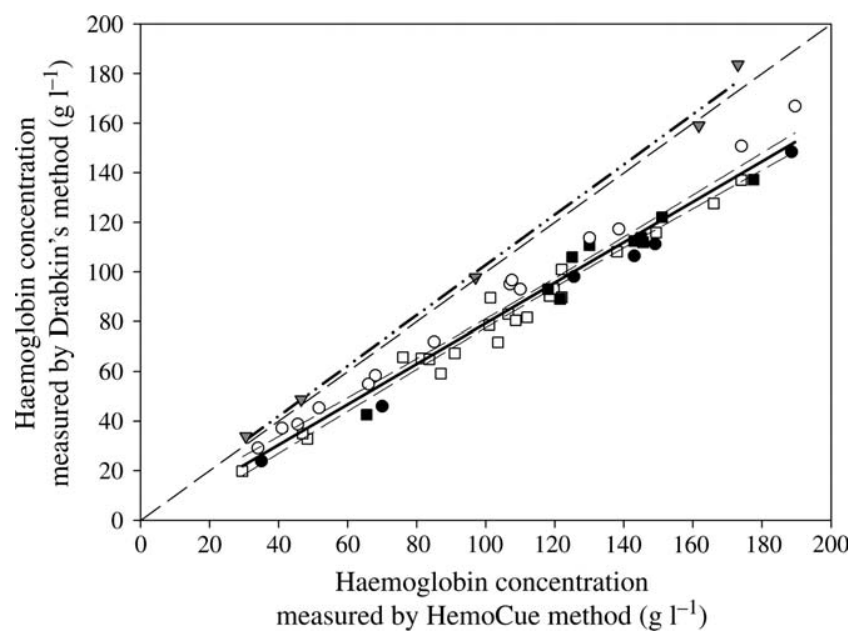

FIG. 1. Comparison of haemoglobin concentrations measured by the Drabkin ([Hb $]_{\text {DRAB }}$ ) and HemoCue ([Hb $]_{\text {HEMO }}$ ) methods for the blood of Oncorhynchus nerka $(\square)$, Oncorhynchus tshawytscha $(\square)$, human $(\nabla)$, Thunnus orientalis $(\bigcirc)$ and Scomber japonicus $(\mathbf{O})$. Data for $[\mathrm{Hb}]_{\text {HEMO }}$ are following a cuvette incubation period of 9-11 min. The curve for human blood (-..) is described by $y=1 \cdot 013 x+1 \cdot 503$ $\left(r^{2}=0.994\right)$ and is not different from the line of equality $(--)$. The curve for the combined fish species (solid line) is described by $y=0 \cdot 815 x-2 \cdot 198$ (---, either side of the regression are $95 \% \mathrm{CI}$ ). 


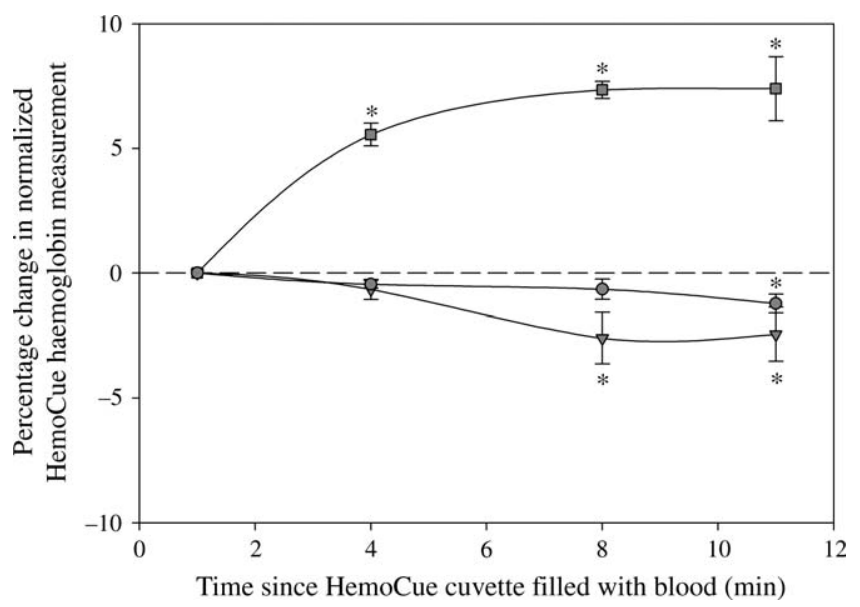

FIG. 2. Relative changes in the haemoglobin reading from the HemoCue analyser during the first 11 min following filling the HemoCue cuvette with blood (means \pm s.E.): pooled data for Oncorhynchus tshawytscha and Oncorhynchus nerka $(\square)$, pooled data for Thunnus orientalis and Scomber japonicus $(\mathrm{O})$ and human blood $(\nabla)$. Note the large fluctuation in measurements for O. tshawytscha and O. nerka blood compared with measurements from all other species. *, significant differences from the initial value at 1 min (repeated measures ANOVA with Dunnett's post hoc test).

This study demonstrated that the HemoCue correctly measured [Hb] of human blood but not fish blood. Because of the systematic nature of the overestimation for fish blood, however, it is possible to accurately calibrate the HemoCue device with the Drabkin method and subsequently use the HemoCue for field studies.

The reason for the discrepancy between the HemoCue and Drabkin methods is unclear. In humans, carboxyhaemoglobin has been reported to cause incorrect measurements of $[\mathrm{Hb}]$ from the HemoCue, possibly because carboxyhaemoglobin may be relatively resistant to conversion into azidemethaemoglobin (Shepherd et al., 2000), which is the required endpoint of the reaction occurring in the HemoCue cuvettes. It is unlikely that the presence of carboxyhaemoglobin was the cause of the overestimation of fish $[\mathrm{Hb}]$ by the HemoCue in the present study. Levels of carboxyhaemoglobin in fishes are generally negligible except when exposed to environments of high carbon monoxide, such as those with intense motorboat traffic (Kempinger et al., 1998). The fishes used in the present study were obtained from vastly different locations, including from fresh water and sea water, none of which were exposed to significant motorboat traffic or carbon monoxide emissions.

Perhaps the most logical explanation for the overestimation of fish [Hb] by the HemoCue is that fish erythrocytes are nucleated. The HemoCue was designed for use on the mammalian erythrocyte that lacks a nucleus. The presence of a nucleus and associated proteins in fish erythrocytes may result in light scattering and thus may be responsible for the disparity in the HemoCue measurements. As far as is known, the role of cell nucleation on HemoCue functionality has not been considered. Even so, this suggestion is consistent with unpubl. obs. on nucleated avian erythrocytes; a $15 \cdot 3 \%$ or $25 \mathrm{~g}^{-1}$ 
overestimation of [Hb] was reported for the budgerigar Melopsittacus undulatus using the HemoCue (Eklom \& Lill, 2006), with a range of $0-25 \%$ or $0-54 \mathrm{~g}^{-1}$ for an unspecified range in [Hb]. Over the range in fish $[\mathrm{Hb}]$ of $20-160 \mathrm{~g}^{-1}$ examined in the present study, overestimates by the HemoCue of $22-50 \%$ or 9.9-36.0 $\mathrm{g} \mathrm{l}^{-1}$ were demonstrated.

Assuming that the four fish species examined in the present study are representative of fishes in general, it is possible to use the equation in Fig. 1 to interpolate the possible errors in $[\mathrm{Hb}]$ that have been reported for previous studies of fishes that have used the HemoCue (Table I). Overestimations in [Hb] of 10 $23 \mathrm{~g} \mathrm{l}^{-1}(21-24 \%)$ are predicted across these studies (Alvarez-Pellitero \& Pinto, 1987; Schmitt et al., 2005, 2007; Ek et al., 2006). Furthermore, [Hb] is often used to calculate other haematological variables, like mean cell haemoglobin concentration (MCHC), and so these will also be reduced by a similar percentage. For example, [Hb] measured by the HemoCue and haematocrit (Hct) for European flounder Platichtys flesus (L.) were reported as $73 \mathrm{~g}^{-1}$ and $25 \%$, respectively (Ek et al., 2006), which would generate a MCHC of $292 \mathrm{~g}^{-1}$ $\left[\mathrm{MCHC}=[\mathrm{Hb}] \cdot\left(\mathrm{Hct} \cdot 100^{-1}\right)^{-1}\right]$. After correcting $[\mathrm{Hb}]$ to $57 \mathrm{~g}^{-1}$ (Fig. 1; equation), $\mathrm{MCHC}$ is reduced to $228 \mathrm{~g}^{-1}$.

It is not evident what caused the unusual variance in $[\mathrm{Hb}]_{\text {HEMO }}$ for $O$. nerka and $O$. tshawytscha for the same blood sample with increasing cuvette incubation time (Fig. 2). The fishes were sexually mature and were migrating to their spawning grounds at capture. Salmonids at this stage of their life cycle undergo many profound haematological changes, including elevated levels of thyroid hormone, gonadal steroid hormone, plasma insulin and cortisol (Leatherland et al., 1989; Kakizawa et al., 1995). The use of a double wavelength measuring method by the HemoCue appropriately accounts for changes in turbidity that may arise from such things as hyperlipemia and the presence of large immunoglobulins (von Schenck et al., 1986; Oeseburg \& Kwant, 1989), yet there are clearly characteristics of sexually mature salmonid blood that cause aberrations in the HemoCue measurements. To minimize variability under these circumstances, it is necessary to monitor and regulate the incubation period allowed for each HemoCue cuvette prior to its measurement. The present study suggests that cuvette incubation periods of $8-11 \mathrm{~min}$ are required for the most consistent results, presumably because it allows complete conversion of haemoglobin into azidemethaemoglobin.

The present findings should encourage the use of hand-held [Hb] analysers under field conditions to provide a rapid indication of the physiological status of individual fishes. With regards to the HemoCue, the calibration equation for fishes (Fig. 1) may be appropriate for most fish species, but prior calibration of the $[\mathrm{Hb}]$ values against the Drabkin method when a new fish species is to be examined is encouraged. Indeed, there were signs of interspecific variability in the present study, with the overestimate of $[\mathrm{Hb}]$ by the HemoCue tending to be less for $T$. orientalis than for the other fish species, but this requires further investigation. There are several medical $[\mathrm{Hb}]$ analysers on the market. If the results for the HemoCue apply to other models, then these analysers could similarly be calibrated for use on fish blood, but given the diversity in the methods used by these analysers to calculate [Hb], calibration equations may well be brand-specific. 


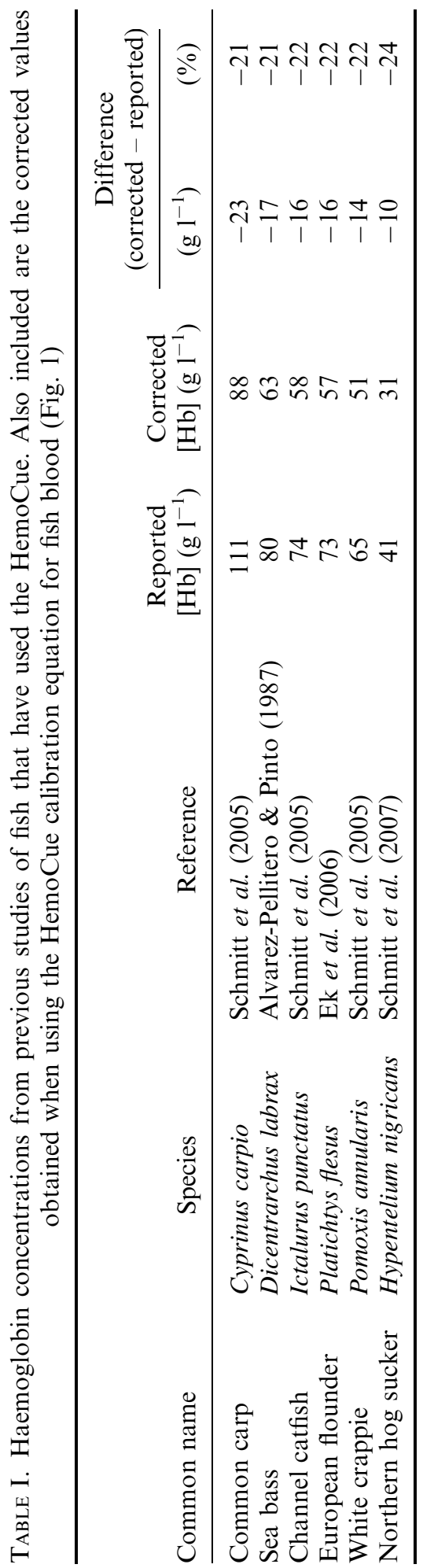


Appreciation is extended to J. Baldwin and R. Wells for constructive discussions relating to this work. B. Block is thanked for allowing access to tuna and mackerel blood. T.D.C. was supported by a Killam Postdoctoral Fellowship. Partial funding for this study was provided by NSERC Discovery and Strategic Grants to S.G.H. and A.P.F. Experiments were conducted in accordance with the Animal Care Protocol of the University of British Columbia (A05-0424).

\section{References}

Alvarez-Pellitero, P. \& Pinto, R. M. (1987). Some blood parameters in sea bass, Dicentrarchus labrax, infected by bacteria, virus and parasites. Journal of Fish Biology 31, 259-261.

Artacho, P., Soto-Gamboa, M., Verdugo, C. \& Nespolo, R. F. (2007). Using haematological parameters to infer the health and nutritional status of an endangered blacknecked swan population. Comparative Biochemistry and Physiology A 147, 1060.

Balasubramaniam, P. \& Malathi, A. (1992). Comparative study of hemoglobin estimated by Drabkin's and Sahli's methods. Journal of Postgraduate Medicine 38, 8-9.

Banbura, J., Banbura, M., Kalinski, A., Skwarska, J., Slomczynski, R., Wawrzyniak, J. \& Zielinski, P. (2007). Habitat and year-to-year variation in haemoglobin concentration in nestling blue tits Cyanistes caeruleus. Comparative Biochemistry and Physiology A 148, 572.

Burness, G. P., McClelland, G. B., Wardrop, S. L. \& Hochachka, P. W. (2000). Effect of brood size manipulation on offspring physiology: an experiment with passerine birds. The Journal of Experimental Biology 203, 3513-3520.

Clark, T. D., Sandblom, E., Cox, G. K., Hinch, S. G. \& Farrell, A. P. (2008). Circulatory limits to oxygen supply during an acute temperature increase in the Chinook salmon (Oncorhynchus tshawytscha). American Journal of Physiology A 295, 16311639. doi: 10.1152/ajpregu.90461.2008

Dacie, J. V. \& Lewis, S. M. (1975). Practical Haematology. London: Churchill Livingstone.

Drabkin, D. L. \& Austin, J. H. (1935). Spectrophotometric studies II. Preparations from washed blood cells; nitric oxide hemoglobin and sulfhemoglobin. Journal of Biological Chemistry 112, 51-65.

Dudaniec, R. Y., Kleindorfer, S. \& Fessl, B. (2006). Effects of the introduced ectoparasite Philornis downsi on haemoglobin level and nestling survival in Darwin's small ground finch (Geospiza fuliginosa). Austral Ecology 31, 88-94.

Ek, H., Dave, G., Nilsson, E., Sturve, J. \& Birgersson, G. (2006). Fate and effects of 2,4,6-trinitrotoluene (TNT) from dumped ammunition in a field study with fish and invertebrates. Archives of Environmental Contamination and Toxicology 51, 244.

Eklom, K. \& Lill, A. (2006). Development of parameters influencing blood oxygencarrying capacity in nestling doves. Ети 106, 283-288.

Frisch, A. \& Anderson, T. (2005). Physiological stress responses of two species of coral trout (Plectropomus leopardus and Plectropomus maculatus). Comparative Biochemistry and Physiology A 140, 317.

Ganong, W. F. (1973). Review of Medical Physiology. Los Altos, CA: Lange Medical Publications.

ICSH (1978). International Committee for Standardization in Haematology. Recommendations for reference method for haemoglobinometry in human blood (ICSH standard EP 6/2: 1977) and specifications for international haemiglobincyanide reference preparation (ICSH standard EP 6/3: 1977). Journal of Clinical Pathology 31, 139-143.

Imsland, A. K., Foss, A., Naevdal, G., Johansen, T., Stefansson, S. O. \& Jonassen, T. M. (2007). New haemoglobin genotypes in Atlantic cod, Gadus morhua: possible relation with growth. Comparative Biochemistry and Physiology A 147, 955.

Kakizawa, S., Kaneko, T., Ogasawara, T. \& Hirano, T. (1995). Changes in plasma somatolactin levels during spawning migration of chum salmon (Oncorhynchus keta). Fish Physiology and Biochemistry 14, 93. 
Kempinger, J. J., Otis, K. J. \& Ball, J. R. (1998). Fish kills in the Fox River, Wisconsin, attributable to carbon monoxide from marine engines. Transactions of the American Fisheries Society 127, 669-672.

Lardi, A. M., Hirst, C., Mortimer, A. J. \& McCollum, C. N. (1998). Evaluation of the HemoCue for measuring intra-operative haemoglobin concentrations: a comparison with the Coulter Max-M. Anaesthesia 53, 349-352.

Lay, P. A. \& Baldwin, J. (1999). What determines the size of teleost erythrocytes? Correlations with oxygen transport and nuclear volume. Fish Physiology and Biochemistry 20, 31-35.

Leatherland, J. F., Down, N. E., Donaldson, E. M. \& Dye, H. M. (1989). Changes in plasma thyroid hormone levels in pink salmon, Oncorhynchus gorbuscha, during their spawning migration in the Fraser River (Canada). Journal of Fish Biology 35, 199-205.

Nyholm, N. E. I., Sawicka-Kapusta, K., Swiergosz, R. \& Laczewska, B. (1995). Effects of environmental pollution on breeding populations of birds in southern Poland. Water, Air, and Soil Pollution 85, 829-834.

O'Brien, E. L., Morrison, B. L. \& Johnson, L. S. (2001). Assessing the effects of haematophagous ectoparasites on the health of nestling birds: haematocrit vs haemoglobin levels in House Wrens parasitized by blow fly larvae. Journal of Avian Biology 32, 73-76.

Oeseburg, B. \& Kwant, G. (1989). Disturbance of the determination of hemoglobin concentration in patients with high leukocyte counts. Clinical Chemistry 35, $515-516$.

von Schenck, H., Falkensson, M. \& Lundberg, B. (1986). Evaluation of "HemoCue", a new device for determining hemoglobin. Clinical Chemistry 32, 526-529.

Schmitt, C. J., Whyte, J. J., Brumbaugh, W. G. \& Tillitt, D. E. (2005). Biochemical effects of lead, zinc, and cadmium from mining on fish in the tri-states district of Northeastern Oklahoma, USA. Environmental Toxicology and Chemistry 24, 1483-1495.

Schmitt, C. J., Whyte, J. J., Roberts, A. P., Annis, M. L., May, T. W. \& Tillitt, D. E. (2007). Biomarkers of metals exposure in fish from lead-zinc mining areas of Southeastern Missouri, USA. Ecotoxicology and Environmental Safety 67, 31-47.

Shepherd, A. P., Steinke, J. M. \& Gong, A. K. (2000). Effects of carboxyhemoglobin on hemoglobinometers. Clinical Chemistry 46, 586-588.

Simmons, P. \& Lill, A. (2006). Development of parameters influencing blood oxygen carrying capacity in the welcome swallow and fairy martin. Comparative Biochemistry and Physiology A 143, 459-468.

Vanzetti, G. (1966). An azide-methemoglobin method for hemoglobin determination in blood. Journal of Laboratory and Clinical Medicine 67, 116-126.

White, C. R. (2003). Allometric analysis beyond heterogeneous regression slopes: use of the Johnson-Neyman technique in comparative biology. Physiological and Biochemical Zoology 76, 135-140. 